\title{
Spinal Cord Stimulation Attenuates Neural Remodeling, Inflammation, and Fibrosis After Myocardial Infarction
}

\author{
Yuxian He, $\mathrm{PhD}^{1}$; Zewei Sun, $\mathrm{PhD}^{1}$; Jiajia Jiang, $\mathrm{MD}^{1}{ }^{\text {; }}$; Xiang Yin, $\mathrm{PhD}^{1}$; \\ Jie Han, $\mathrm{PhD}^{1}$; Yuanyuan Zhang, $\mathrm{PhD}^{2}{ }^{\oplus}$; Liangrong Zheng, $\mathrm{PhD}^{1}$
}

\begin{abstract}
Objectives: Spinal cord stimulation (SCS) is an established neuromodulation method that regulates the cardiac autonomic system. However, the biological mechanisms of the therapeutic effects of SCS after myocardial infarction (MI) remain unclear.

Materials and Methods: Twenty-five rabbits were divided into five groups: SCS-MI (voltage: 0.5 v; pulse width: $0.2 \mathrm{~ms} ; 50 \mathrm{~Hz}$; ten minutes on and 30 minutes off; two weeks; $n=5), \mathrm{MI}(n=5)$, sham SCS-MI (voltage: $0 \mathrm{v}$; two weeks; $n=5)$, sham MI $(n=5)$, and blank control $(n=5)$ groups. MI was induced by permanent left anterior descending artery ligation. SCS-MI and sham SCS-MI rabbits received the corresponding interventions 24 hours after Ml. Autonomic remodeling was evaluated using enzymelinked immunosorbent assay and immunohistochemistry. Inflammation and myocardial fibrosis were assessed using immunohistochemistry, quantitative polymerase chain reaction, hematoxylin and eosin staining, Masson staining, and Western blot.

Results: SCS improved the abnormal systemic autonomic activity. Cardiac norepinephrine decreased after $\mathrm{MI}(p<0.01)$ and did not improve with SCS. Cardiac acetylcholine increased with SCS compared with the MI group $(p<0.05)$. However, no difference was observed between the MI and blank control groups. Growth-associated protein $43(p<0.001)$ and tyrosine hydroxylase $(p<0.001)$ increased whereas choline acetyltransferase $(p<0.05)$ decreased in the Ml group compared with the blank control group. These changes were attenuated with SCS. SCS inhibited inflammation, decreased the ratio of phosphorylated-Erk to Erk $(p<0.001)$, and increased the ratio of phosphorylated-STAT3 to STAT3 $(p<0.001)$ compared with the Ml group. Myocardial fibrosis was also attenuated by SCS.

Conclusions: SCS improved abnormal autonomic activity after Ml, leading to reduced inflammation, reactivation of STAT3, and inhibition of Erk. Additionally, SCS attenuated myocardial fibrosis. Our results warrant future studies of biological mechanisms of the therapeutic effects of SCS after MI.
\end{abstract}

Keywords: Autonomic remodeling, inflammation, myocardial fibrosis, myocardial infarction, spinal cord stimulation

Conflict of Interest: The authors reported no conflict of interest.

\section{INTRODUCTION}

In the past few decades, progress has been made in the treatments for acute myocardial infarction (MI), including percutaneous coronary intervention, antithrombotic therapy, and secondary prevention, which has led to a decrease in the incidence and mortality of acute MI in many developed countries. ${ }^{1,2}$ However, a systematic review suggested that although the mortality and morbidity after acute MI continued to decline from the past to the recent study period, the relative risks for all-cause death and adverse cardiovascular outcomes (such as recurrent $\mathrm{Ml}$ and cardiovascular death) were at least $30 \%$ higher than those in the general population at one to three years and three to five years after $\mathrm{Ml}^{3}{ }^{3}$ Therefore, $\mathrm{Ml}$ is still one of the most important diseases that affect humans, and new treatments are needed to treat MI.

Many researchers have suggested that hypertension, $\mathrm{Ml}$, heart failure (HF), and many other primary cardiovascular diseases are associated with dysfunction of the autonomic nervous system (ANS). ${ }^{4,5}$ The management and prevention of cardiovascular autonomic dysfunction may be beneficial in the treatment for MI to improve the prognosis. Spinal cord stimulation (SCS) is a reliable and effective method for neuromodulation that has been used to treat chronic intractable pain for decades. ${ }^{6-8}$ Numerous studies have demonstrated positive effects of SCS on a variety of ischemic

Address correspondence to: Liangrong Zheng, Department of Cardiology, the First Affiliated Hospital, College of Medicine, Zhejiang University, No. 79 Qingchun Road, Hangzhou, 310,003 China. Email: 1191066@zju.edu.cn

1 Department of Cardiology, The First Affiliated Hospital, Zhejiang University School of Medicine, Hangzhou, China; and

2 Department of Cardiology, The Affiliated Hospital of Hangzhou Normal University, Hangzhou, Zhejiang, China

For more information on author guidelines, an explanation of our peer review process, and conflict of interest informed consent policies, please see the journal's Guide for Authors.

Source(s) of financial support: This work was supported by the National Key Research and Development Program of China (grant no. 2016YFC1301003), the National Natural Science Foundation of China (No. 81873484), the National Natural Science Foundation of China Youth Project (No. 82000316), and Zhejiang Provincial Natural Science Foundation (LQ18H020006). 
heart diseases. In patients with refractory angina pectoris who are not suitable for revascularization, SCS improved refractory angina pectoris and refractory microvascular angina pectoris, leading to reduced episodes of angina and the reduced need for short-acting nitrate tablets. ${ }^{9}$ Short-term SCS improved myocardial ischemic tolerance and perfusion reserve in patients with refractory angina pectoris. ${ }^{10}$ Studies have demonstrated that thoracic SCS was safe and feasible for the treatment of HF and improved the symptoms, left ventricular function, and remodeling in severe HF patients. ${ }^{11,12}$ However, a subsequent study did not find evidence to support these findings. ${ }^{13}$ There are additional requirements for studies that evaluate the ideal SCS parameter settings (ie, SCS dose). ${ }^{14}$ Animal studies of ischemic heart disease demonstrated that SCS protected the ischemic myocardium by regulating multiple levels of the cardiac ANS in a reversible, on-demand manner. In a porcine model of HF, SCS induced the remodeling of cardiac sympathetic innervation in the peri-infarct and infarct regions, which improved the left ventricular function. ${ }^{15}$ In a canine model of MI, SCS inhibited the left stellate ganglion neural activity and protected against MIinduced ventricular arrhythmias. ${ }^{16}$ In other studies, SCS mitigated the afferent sensory transduction of the ischemic myocardium and attenuated the ANS feedback control, thereby regulating the sympathetic nerve input of the ischemic heart and providing a stable environment for the cardiomyocytes. ${ }^{17,18}$

In recent years, researchers have made efforts to optimize electronic devices and stimulation parameter settings for SCS. ${ }^{19,20}$ However, the biological mechanisms of the therapeutic effects of SCS on the ischemic heart have not been fully elucidated. It is important to determine these mechanisms to achieve highly selective and effective treatment of SCS. ${ }^{19}$ The aim of the present study was to detect the effects of SCS on autonomic activity, inflammation, and cardiac fibrosis after $\mathrm{Ml}$ and to provide a theoretical basis for future research of the biological mechanisms of SCS treatment for MI.

\section{MATERIALS AND METHODS}

\section{SCS System Implantation}

The present study was conducted in accordance with the Guidelines for the Care and Use of Laboratory Animals published by the US National Institutes of Health (publication No. 85-23, revised 1996). This study was approved by the institutional review board of Zhejiang Laboratory Animal Center (approval no. ZJCLA-IACUC20010011). Thirty-five adult male New Zealand rabbits, weighing approximately two kg, were bred and supplied by the Zhejiang Laboratory Animal Center. All rabbits were randomly divided into five groups: SCS-MI (voltage: $0.5 \mathrm{v}$; pulse width: $0.2 \mathrm{~ms} ; 50 \mathrm{~Hz}$; ten minutes on and 30 minutes off; two weeks; $n=7)$, MI $(n=7)$, sham SCS-MI (voltage: 0 v; two weeks; $n=7)$, sham MI $(n=7)$, and blank control ( $n=7$ ) groups (Fig. 1C). Animals in SCS-MI and sham SCS-MI groups were anesthetized with $3 \%$ pentobarbital sodium $(30 \mathrm{mg} /$ $\mathrm{kg}$ ) and, if rabbits remained uneasy, pentobarbital sodium supplements $(7.5 \mathrm{mg} / \mathrm{kg})$. Anesthesia was administered until the grasping response of the rabbits disappeared. All rabbits lay curled up on their sides on the operating table to increase the distance between lumbar vertebrae. Following the preparation of the surgical area, the skin and the vertebral muscles were bluntly dissected, and the L3-4 intervertebral ligaments were carefully exposed and incised. A sterile SCS electrode (Octrode; model 3,778 to 75; Medtronic Inc, Parkway, MN) was slowly inserted into the epidural space under $x$-ray visualization, and the distal end of the electrode was fixed at the level of T3-4 (Fig. 1a). The puncture metal core was removed, and the proximal end of the electrode was connected to the electrical stimulator (PrimeADVANCED ${ }^{\text {TM }}$ Multi-program Neurostimulator for Spinal Cord Stimulation; model 37,702; Medtronic Inc, Parkway). The electrical stimulator and wire outside the epidural space were embedded in the sterile subcutaneous sac, which was prepared in advance.

\section{Establishment of MI}

Two weeks after the SCS systems were implanted, MI was induced using methods described previously. ${ }^{21}$ Animals from SCSMI, MI, sham SCS-MI, and sham MI groups were anesthetized. A tracheotomy was performed on these animals to connect the respiratory tract to a small animal ventilator. The following breathing parameters and equipment were used: tracheal catheter: ID 2.5 $\mathrm{mm}$; respiratory rate: $35 / \mathrm{min}$; and tidal volume: $10 \mathrm{~mL} / \mathrm{kg}$. A left lateral thoracotomy was performed through the third intercostal space. In the SCS-MI, MI, and sham SCS-MI groups, the pericardium was opened, and the proximal one-third of the left anterior descending (LAD) artery was ligated with a 6-0 sterile silk thread. The pericardium was also opened in the sham Ml group, but the $\mathrm{LAD}$ artery was not ligated.

\section{Electrocardiography}

Animals were anesthetized using $3 \%$ pentobarbital sodium (20 $\mathrm{mg} / \mathrm{kg}$ ) until they were calm, and they were gently fixed on the operating table in a supine position. Electrocardiograms (ECGs) were used to detect MI after 24 hours of the LAD ligation. MI was confirmed on the basis of changes in the color of myocardium after LAD ligation and ST segment elevation $(>0.2 \mathrm{mV})$ and $\mathrm{T}$ wave inversion on ECG lead II. All ECG data were acquired using a multichannel electrophysiological recording system (Transonic Scisense Inc., London, ON, Canada) (Fig. 1b).

\section{Collection of Plasma and Heart Samples}

Blood samples and cardiac tissues were collected at the end of the experiment (two weeks after Ml). Blood was immediately centrifuged at $3000 \mathrm{~g}$ for 15 minutes, and the supernatant was transferred to a new freezing tube and stored at $-80^{\circ} \mathrm{C}$. Each heart sample was divided into two parts for later experiments.

\section{Effects of SCS on Neurotransmitters}

To investigate the effect of SCS on ANS activity, the concentrations of norepinephrine (NE) and acetylcholine (Ach) in the plasma and cardiac tissue and the concentration of plasma epinephrine (E) were detected using rabbit enzyme-linked immunosorbent assay (ELISA; Fankewei Co., Ltd., Shanghai, China) in accordance with the manufacturer's instructions. Protein levels were calculated using standard curves. Tissue ELISA measurements were normalized according to the protein content of the homogenates $\left(\mu \mathrm{g} \times \mathrm{mL}^{-1} / \mu \mathrm{g}\right.$ protein for Ach; $\mathrm{ng} \times \mathrm{mL}^{-1} / \mu \mathrm{g}$ protein for NE). Each experiment was replicated independently at least three times.

\section{Effects of SCS on the Sympathetic and Parasympathetic Nerve Fibers}

As previously reported, we evaluated the redistribution of sympathetic and parasympathetic nerve fibers in the peri-infarct zone of heart tissue two weeks after MI using immunohistochemistry. ${ }^{22}$ In the present study, peri-infarct zones (the 2-mm area adjacent to the pathological infarct) in cardiac tissues from the SCS-MI, MI 
a

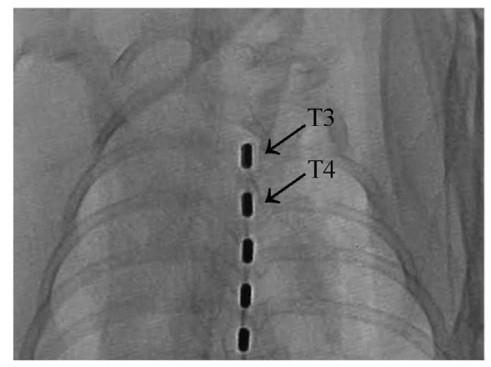

b

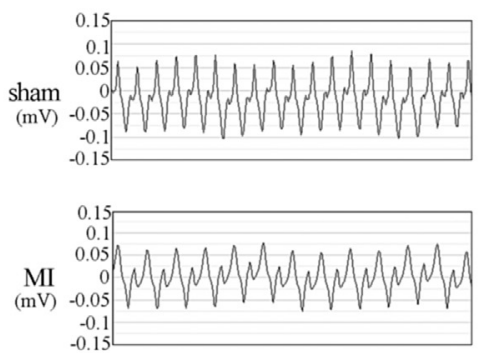

C

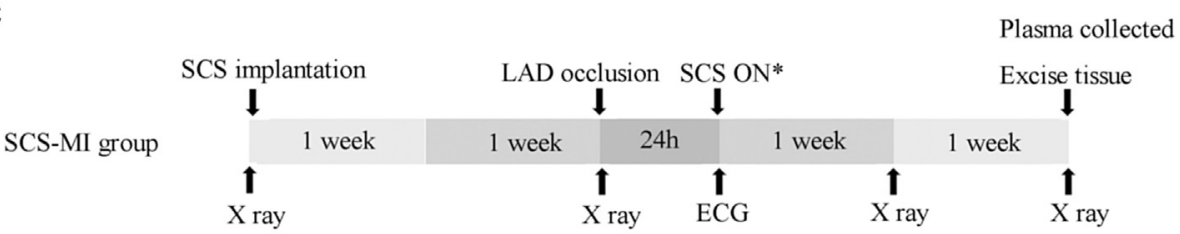

\begin{tabular}{|c|c|c|c|c|c|}
\hline \multirow{4}{*}{ MI group } & \multirow{2}{*}{\multicolumn{4}{|c|}{ LAD occlusion }} & Plasma collecte \\
\hline & & & & & Excise tissue \\
\hline & 1 week & 1 week & $24 \mathrm{~h}$ & 1 week & 1 week \\
\hline & & & & & \\
\hline
\end{tabular}

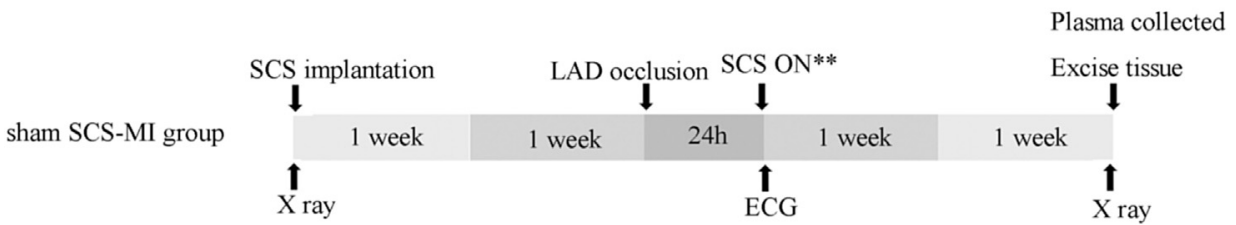

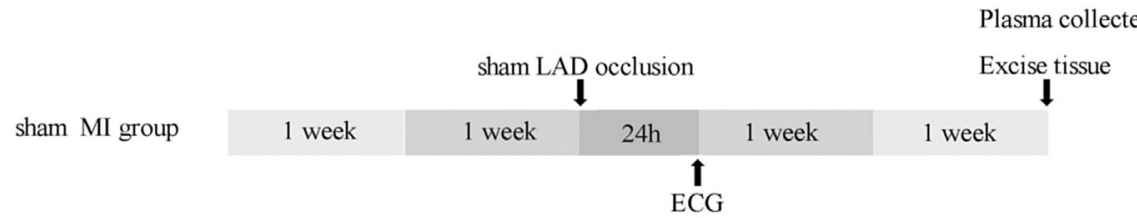

\begin{tabular}{|c|c|c|c|c|c|}
\hline \multirow[b]{2}{*}{ blank control group } & & & & & $\begin{array}{l}\text { Plasma collected } \\
\text { Excise tissue } \\
\downarrow\end{array}$ \\
\hline & 1 week & 1 week & $24 \mathrm{~h}$ & 1 week & 1 week \\
\hline
\end{tabular}

Figure 1. a. Perspective image of the location of the two octopole leads at T3-4 position (b) electrocardiogram before and after surgery to induce myocardial infarction (c) schematic diagram for the research protocol flow chart. *SCS parameters were set as follows: voltage: $0.5 \mathrm{v}$; pulse width: $0.2 \mathrm{~ms} ; 50 \mathrm{~Hz}$; ten minutes on and 30 minutes off; 2 weeks. ${ }^{*}$ SCS parameters were set as follows: voltage: 0 v; pulse width: $0.2 \mathrm{~ms} ; 50 \mathrm{~Hz}$; ten minutes on and 30 minutes off; 2 weeks.

and sham SCS-MI groups as well as the same zone in sham MI and blank control groups were analyzed using methods described previously. ${ }^{23}$ Adjacent sections (midpoint between LAD ligation site and apex) of $5-\mu \mathrm{m}$ thickness were made perpendicular to the long axis of the heart. The following primary antibodies were used: tyrosine hydroxylase (TH; 1:500; Abcam, Cambridge, UK), growthassociated protein 43 (GAP43; 1:500; Abcam), and choline acetyltransferase (ChAT; 1:500; Abcam). Three random fields of view were counted per section; two sections were analyzed in each heart, and at least three hearts were examined in each group. The images were analyzed using the ImageJ software Image-Pro Plus 5.0 (Media Cybernetics, Inc., Bethesda, MD).

\section{Effects of SCS on MI-Induced Inflammation}

According to previous studies, ANS regulates inflammation. To study the effects of SCS on inflammation, we evaluated macrophage infiltration and expression of inflammatory factors. The expression of inflammatory factors was evaluated using reverse transcription polymerase chain reaction (RT-PCR). Briefly, total RNA was extracted from the peri-infarct myocardial samples using TRIzol 
Table 1. Primer Sequences for Rabbits.

\begin{tabular}{|c|c|}
\hline Gene Name & Primer Sequence \\
\hline NGF & 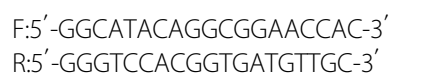 \\
\hline $\mid \mathrm{L}-1 \beta$ & $\begin{array}{l}\text { F:5'-CACCAGTGAGATGATGGCTTAC-3' } \\
\text { R:5'-GAGCCACAACGACTGACAAGA-3' }\end{array}$ \\
\hline IL-6 & $\begin{array}{l}\text { F:5'-AAGGCTGATAGAAGAAGACGGA-3 } \\
\text { R:5'-TTCTTCGTCACTCCTGAACTTG-3' }\end{array}$ \\
\hline TNFa & 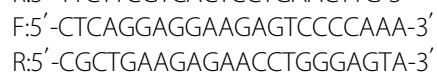 \\
\hline GAPDH & 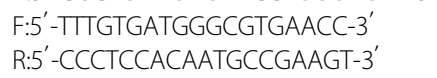 \\
\hline
\end{tabular}

F: forward; R: reverse; GAPDH: glyceraldehyde-phosphate dehydrogenase; NGF: nerve growth factor; RT-PCR: reverse transcription polymerase chain

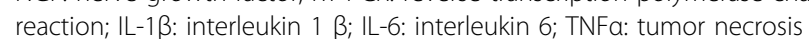
factor $a$.

reagent and RNA extraction kit (Invitrogen, Paisley, United Kingdom; Thermo Fisher Scientific, Inc., Waltham, MA). The purity of RNA samples was assessed using ultraviolet absorption at 260 and $280 \mathrm{~nm}$. The samples were reverse-transcribed using a PrimeScript RT reagent kit (TaKaRa Biotechnology [Dalian] Co., Ltd., Beijing, China). Real-time PCR was performed using SYBR Premix Ex Taq (TaKaRa Biotechnology [Dalian] Co., Ltd., Beijing, China) and a Mastercycler EP realplex detection system (Eppendorf, Hamburg, Germany). Gene expression was analyzed using the $2^{-\Delta \Delta C T}$ method described previously. ${ }^{24}$ Table 1 summarizes the primer sequences used in this study. Macrophage infiltration was assessed using immunohistochemistry and CD68 (1:500; Abcam). Additionally, immunofluorescence co-localization was performed using CD68 (1:500; Abcam) and nerve growth factor (NGF; 1:50; Santa Cruz Biotechnology, Inc., Dallas, TX). NGF levels (1:200; Santa Cruz Biotechnology, Inc) were detected using Western blot.

\section{Effects of SCS on Erk and STAT3 Phosphorylation}

Previous studies have demonstrated that the Erk and STAT3 pathways were involved in the regulation of inflammation by the ANS. We studied the effects of SCS on the Erk and STAT3 phosphorylation using Western blot. Briefly, peri-infarct areas of the heart tissues were homogenized on ice in an ultrasonic machine. The total proteins and protein concentrations were detected using the protein extraction kit (Beyotime Institute of Biotechnology, Haimen, Jiangsu, China) and BCA protein assay kit (Beyotime Institute of Biotechnology), respectively. The primary antibodies used in this study were anti-STAT3 (1:1500; Cell Signaling Technology, Inc, Danvers, MA), anti-p-STAT3 (1:1000; Cell Signaling Technology, Inc), anti-Erk (1:1000; Cell Signaling Technology, Inc), anti-p-Erk (1:1000; Cell Signaling Technology, Inc), and $\beta$-actin (1:3000, Proteintech Group, Inc, Rosemont, IL). All results were analyzed using the ImageJ software Image-Pro Plus 5.0.

\section{Effects of SCS on MI-Induced Myocardial Fibrosis}

The effects of SCS on Ml-induced myocardial fibrosis were evaluated using the Masson's trichrome stain, hematoxylin and eosin (HE) stain, and Western blot. As previously mentioned, adjacent sections (midpoint between LAD ligation site and apex) of $5-\mu \mathrm{m}$ thickness were prepared perpendicular to the long axis of the heart and stained with Masson's trichrome stain. ${ }^{25}$ The ratio of the fibrotic area to the total area in each image was calculated using the Image-Pro Plus 5.0 software. For HE staining, the slices were flattened in warm water at about $42{ }^{\circ} \mathrm{C}$ and baked to prepare paraffin tissue sections. These sections were conventionally dewaxed with xylene solution, dehydrated with gradient alcohol, and stained with hematoxylin for ten minutes at room temperature. After the sections were washed and differentiated in hydrochloric acid alcohol solution for several seconds, the sections were washed again and reacted with eosin for 30 seconds. Finally, the slices were rehydrated with gradient alcohol followed by moderate color development and sealed. Western blot was performed as described previously. The primary antibodies used in this study were antiTGF $\beta$ (1:1000; Abcam), anti-CTGF (1:1000; Abcam), anti-aSMA (1:500; Proteintech Group, Inc), and $\beta$-actin (1:3000; Proteintech Group, Inc). All results were analyzed using the ImageJ software Image-Pro Plus 5.0.

\section{Cell Cultures and Interventions}

RAW 264.7 macrophage cell lines were purchased from the American Type Culture Collection (Manassas, VA), cultured using Dulbecco's modified Eagle medium (5.5 mM; Gibco; Thermo Fisher Scientific, Inc), and supplemented with $10 \%$ fetal bovine serum, 100 $\mathrm{U} / \mathrm{ml}$ of penicillin, and $100 \mu \mathrm{g} / \mathrm{mL}$ of streptomycin at $37{ }^{\circ} \mathrm{C}$ in a humidified atmosphere $\left(5 \% \mathrm{CO}_{2}\right.$ and $95 \%$ air). Lipopolysaccharides (LPS) were purchased from Sigma-Aldrich (cat. no: L2880; Merck KGaA, Darmstadt, Germany), and NE was purchased from SigmaAldrich (cat. no: A0937; Merck KGaA). Different concentrations (0, $0.01,0.1$, or $1 \mu \mathrm{M}$ ) of NE were used to process the LPS-activated (1 $\mu \mathrm{g} / \mathrm{mL}$ ) RAW264.7 cell lines for 12 hours, while using $1 \mu \mathrm{M}$ NE to process the RAW264.7 cell lines without LPS activation. RAW 264.7 cells were collected for analysis.

\section{Inhibitors and Activators}

Colivelin (a STAT3 activator) was purchased from Santa Cruz Biotechnology. U0126 (an Erk inhibitor) and Stattic (a STAT3 inhibitor) were purchased from Selleck Chemicals (Houston, TX). RAW264.7 cells were pretreated with different activators and inhibitors for 30 minutes, followed by processing of these cells (1 $\mu \mathrm{g} / \mathrm{mL})$ with NE ( $1 \mu \mathrm{M})$ for 12 hours. RAW 264.7 cells were collected for analysis.

\section{Statistical Analysis}

Data from the three independent repetitions of experiments were expressed as means \pm standard deviations (SDs). One-way analysis of variance (ANOVA) followed by Tukey's multiple comparisons test were used for multiple-group comparisons. GraphPad Prism (version 5.0; GraphPad software Inc, San Diego, CA) was used for analysis. $p$ values $<0.05$ were considered statistically significant.

\section{RESULTS}

Thirty-five rabbits were included in this study: the SCS wire fell off during x-ray examination two weeks after MI in two rabbits; one died five days after sham $\mathrm{Ml}$, probably because of a respiratory infection; one suffered paralysis of both posterior limbs after SCS system implantation; and three died three to seven days after MI. Five freely moving rabbits, who spontaneously recovered from two surgeries, were selected from each group for analysis. Until the end of the experiment, none of these animals died as a result of MI. 
a

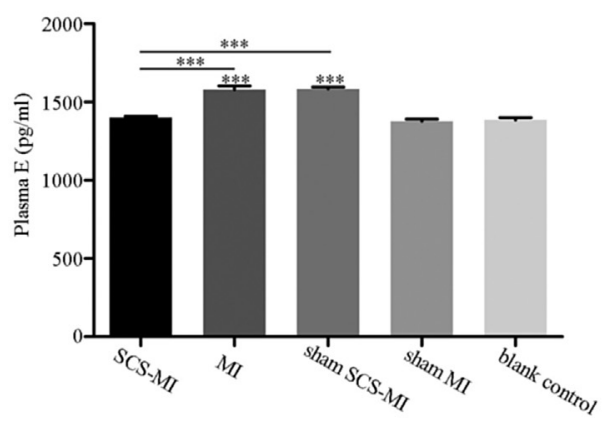

b

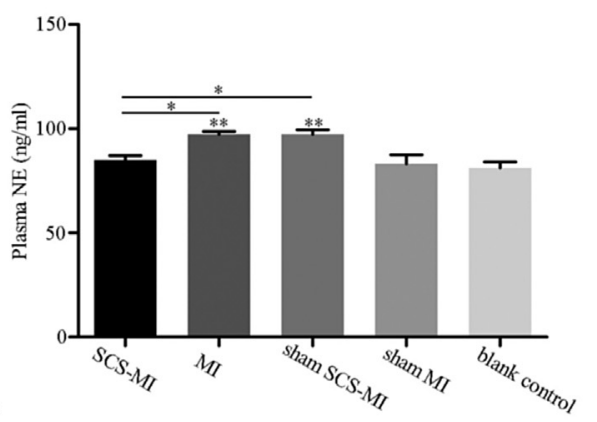

C

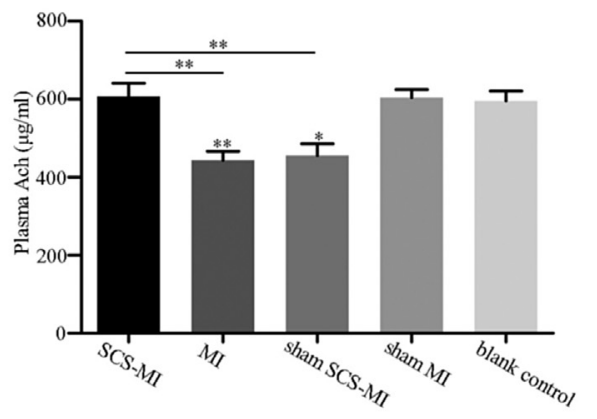

d

$d$

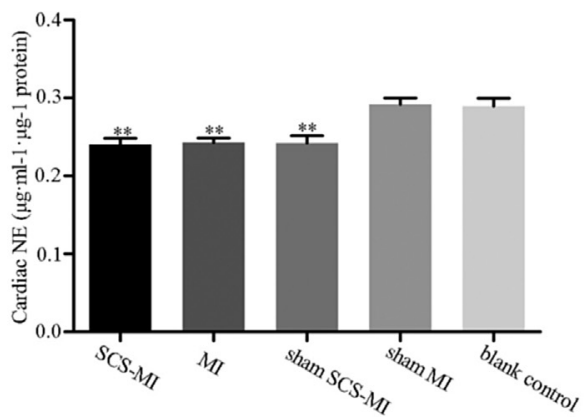

e

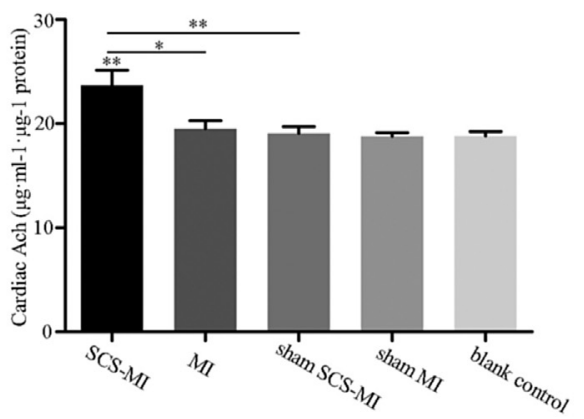

Figure 2. Effect of SCS on systemic and cardiac neurotransmitters. a-c. Plasma E, NE, and Ach levels measured using ELISA. d-e. Cardiac NE and Ach levels in the periinfarct area three weeks after $\mathrm{Ml}$ among the five groups mentioned previously. $\mathrm{E}$, adrenaline; $\mathrm{NE}$, norepinephrine; Ach, acetylcholine; MI, myocardial infarction; ELISA, enzyme-linked immunosorbent assay. Data from three independent experiments were presented as means \pm standard deviations. One-way ANOVA followed by Tukey's multiple comparisons test were used for multiple-group comparisons $\left({ }^{*} p<0.05,{ }^{* *} p<0.01,{ }^{* * *} p<0.001\right)$.

\section{Effects of SCS on Neurotransmitters}

Plasma NE (Fig. 2b; $p<0.01$ ) and E (Fig. 2a; $p<0.001$ ) levels increased in the Ml group compared with the blank control group, and this increase was attenuated by SCS (Fig. 2a,b; $p<0.05$ and $p<0.001$, respectively). Conversely, plasma Ach levels decreased in the Ml group (Fig. 2c; $p<0.01$ ) compared with the blank control group, and this decrease was mitigated by SCS (Fig. 2c; $p<0.01$ ). Importantly, different neurotransmitters were found in the heart and plasma. Cardiac NE levels decreased in the Ml group (Fig. 2d; $p<0.01)$ compared with the blank control group, which was not affected by SCS. Additionally, cardiac Ach levels increased in the SCS-MI group compared with the MI group (Fig. 2e; $p<0.05$ ). However, there was no difference in the cardiac Ach levels between the Ml and blank control groups (Fig. 2e). Generally, there was no difference between the MI and sham SCS-MI groups or between the sham Ml and blank control groups.

\section{Effects of SCS on the Sympathetic and Parasympathetic Nerve Fibers}

The density of GAP43 (Fig. 3a,c; $p<0.001$ ) and TH-positive nerve fibers (Fig. 3a,d; $p<0.001$ ) was higher in the Ml group compared with the blank control group. However, the density of GAP43 (Fig. 3a,c; $p<0.001$ ) and TH-positive nerve fibers (Fig. 3a,d; $p<0.001$ ) was lower in the SCS-MI group compared with the MI group. Previous studies have demonstrated that ChAT catalyzes the synthesis of Ach and can be used to identify putative cholinergic neurons. Therefore, we used ChAT to detect the cholinergic neurons. Compared with the blank control group, the MI group had a lower density of ChAT-positive nerve fibers (Fig. 3a,b; $p<0.05$ ).
However, this decrease was attenuated in comparison with the SCS-MI group (Fig. 3a,b; $p<0.01$ ). There was no difference between the MI and sham SCS-MI groups or between the sham MI and blank control groups.

\section{Effects of SCS on MI-Induced Inflammation}

CD68 levels were higher in the SCS-MI (Fig. 4a,b; $p<0.05$ ) and MI (Fig. $4 \mathrm{a}, \mathrm{b} ; p<0.001$ ) groups than in the blank control group. However, CD68 level was lower in the SCS-MI group than in the MI group (Fig. 4a,b; $p<0.01$ ). Tumor necrosis factor (TNF)-a, interleukin (IL)-6, and IL-1 $\beta$ were also higher in the SCS-MI (Fig. 4c-e; $p<0.001, p<0.01$, and $p<0.05$, respectively) and MI (Fig. 4c-e; $p<0.001, p<0.001$, and $p<0.001$, respectively) groups than in the blank control group. However, the levels of TNF- $\alpha$, IL-6, and IL-1 $\beta$ were lower in the SCS-MI group than in the MI group (Fig. $4 \mathrm{c}-\mathrm{e}$; $p<0.001, p<0.001$, and $p<0.001$, respectively). The mRNA and NGF levels were lower in the SCS-MI group than in the MI group (Fig. $4 \mathrm{f}-\mathrm{h} ; p<0.01$ and $p<0.001$, respectively). However, there was no difference between the MI and sham SCS-MI groups or between the sham MI and blank control groups. In addition, immunofluorescence colocalization demonstrated that NGF colocalized with $\mathrm{CD}^{+} 8^{+}$inflammatory cells (Fig. 4i).

\section{Effects of SCS on the Erk and STAT3 Phosphorylation}

Compared with the blank control group, the SCS-MI and MI groups had higher ratios of phosphorylated-Erk to total Erk (Fig. 5a,b; $p<0.01$ and $p<0.001$ ). However, the increase in the ratio of the SCS-MI group was inhibited compared with the MI group (Fig. 5a,b; $p<0.001$ ). Compared with the blank control 


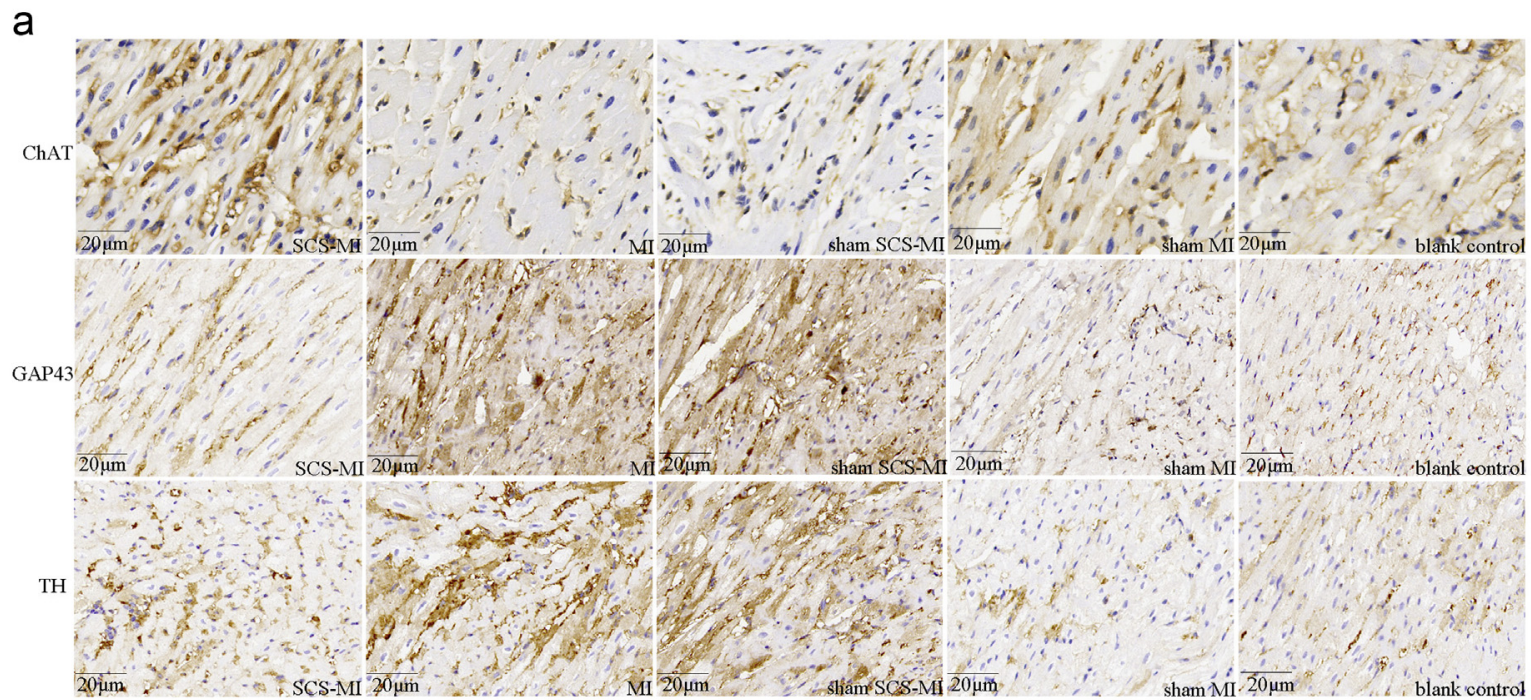

b

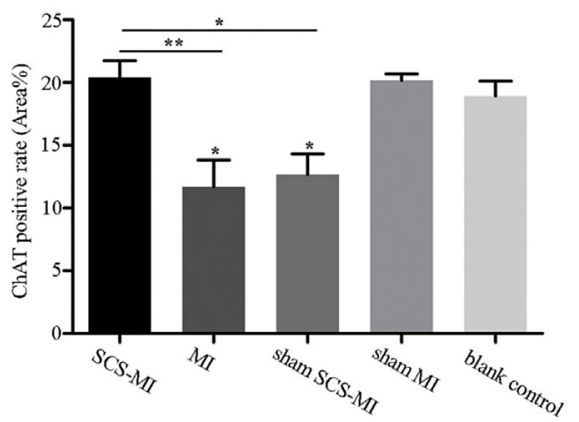

c

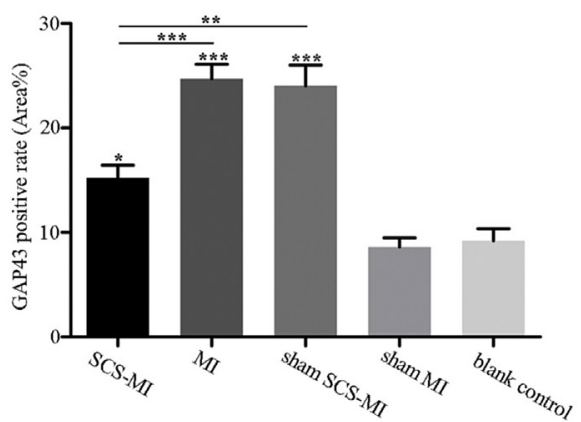

d

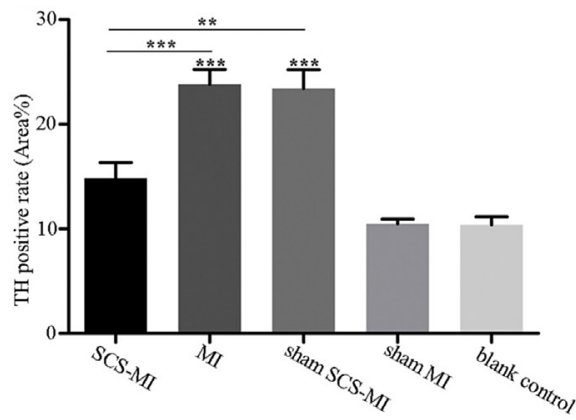

Figure 3. Effects of SCS on regional sympathetic and parasympathetic nerve fibers. a. Representative immunohistochemistry photomicrographs of ChAT, GAP43, and TH in the peri-infarct area two weeks after Ml from the five groups mentioned previously. b-d. Quantitative analysis of a. ChAT, choline acetyltransferase; TH, tyrosine hydroxylase; GAP43, growth-associated protein 43; MI, myocardial infarction. Three random fields of view were counted per section; two sections were evaluated from each heart, and three hearts were evaluated from each group. Data from three independent experiments were presented as means \pm standard deviations. One-way ANOVA followed by Tukey's multiple comparisons test were used for multiple-group comparisons $\left({ }^{*} p<0.05,{ }^{* *} p<0.01,{ }^{* * *} p<0.001\right)$. [Color figure can be viewed at www.neuromodulationjournal.org]

group, the SCS-MI and $\mathrm{MI}$ groups had lower ratios of phosphorylated-STAT3 to total STAT3 (Fig. $5 \mathrm{c}, \mathrm{d} ; p<0.01$ and $p<0.001)$. The decrease in this ratio of the SCS-MI group was attenuated compared with the Ml group (Fig. $5 c, d ; p<0.001$ ). No differences were observed between the MI and sham SCS-MI groups and between the sham $\mathrm{Ml}$ and blank control groups.

\section{Effect of SCS on MI-Induced Myocardial Fibrosis}

HE staining (Fig. 6a) did not demonstrate any morphological abnormality of the myocardial cells in the sham Ml or blank control groups. The myocardial cell structure was complete and regular, and the myocardial fibers were arranged in an orderly manner. However, in the MI and sham SCS-MI groups, myocardial cells exhibited significant damage, with irregular morphology, incomplete structure, and disorderly arrangement. The myocardial fibers demonstrated rupture and necrosis, irregular morphology, and incomplete structure. There were also fibroblast proliferation and scar tissue formation. Despite the partial loss of myocardial cells in the SCS-MI group, the morphology and structure of the myocardial cells were better than those in the MI and sham SCS-MI groups.
Masson's trichrome staining demonstrated that there was less myocardial fibrosis in the SCS-MI group than in the MI group (Fig. 6a,b; $p<0.01$ ). Similarly, the levels of CTGF (Fig. $6 \mathrm{c}, \mathrm{d} ; p<$ 0.001), a-SMA (Fig. $6 c, e ; p<0.01$ ), and TGF- $\beta$ (Fig. $6 c$,f; $p<0.001$ ) were lower in the SCS-MI group than in the MI group. There was no difference between the MI and sham SCS-MI groups or between the sham and blank control groups.

\section{DISCUSSION}

These were the main findings of this study: (1) SCS rebalanced the systemic sympathetic and parasympathetic activity in Ml rabbits. However, the decrease in cardiac NE after MI was not affected by SCS. Cardiac Ach was higher in the SCS group than in the MI group, but there was no difference between the $\mathrm{Ml}$ and blank control groups; (2) SCS modified the cardiac sympathetic and parasympathetic nerve fibers after $\mathrm{Ml}$; (3) the increased inflammatory response and Erk phosphorylation as well as decreased STAT3 phosphorylation after MI were mitigated by SCS; and (4) SCS attenuated myocardial fibrosis after MI. 


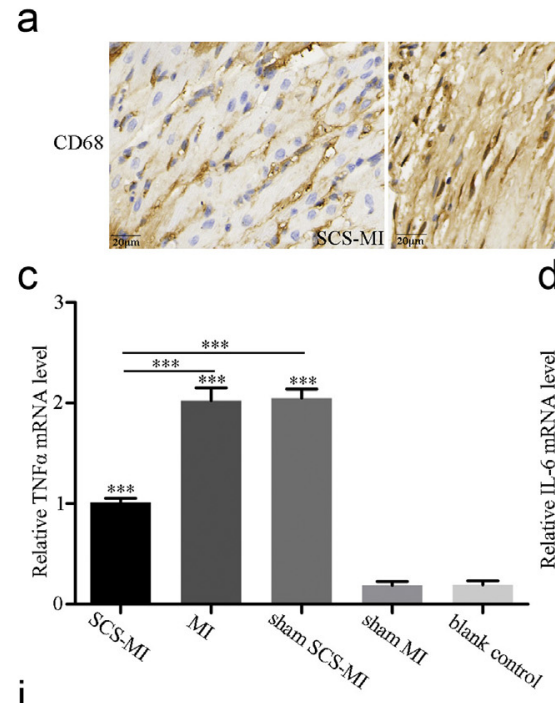

i
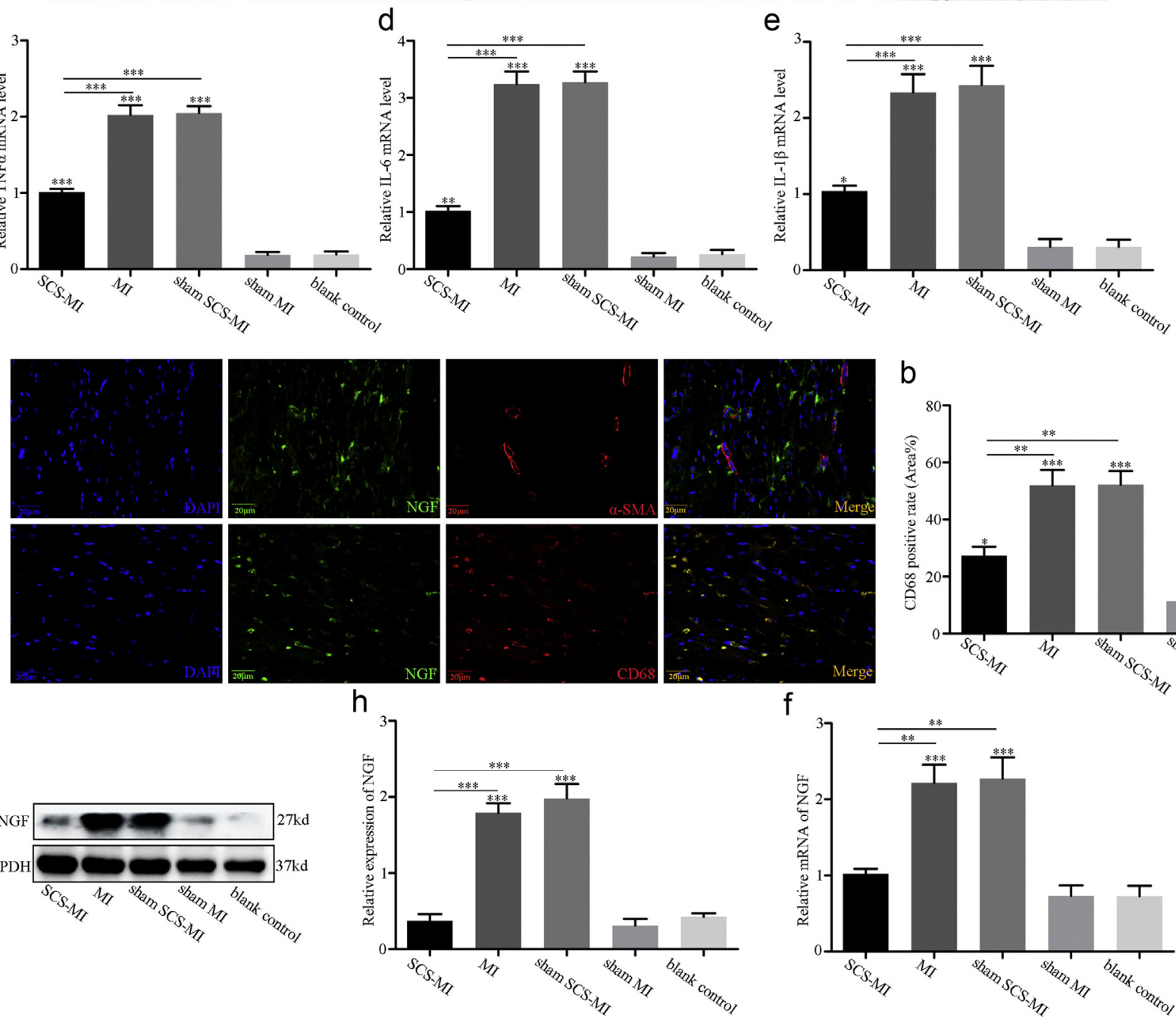

b
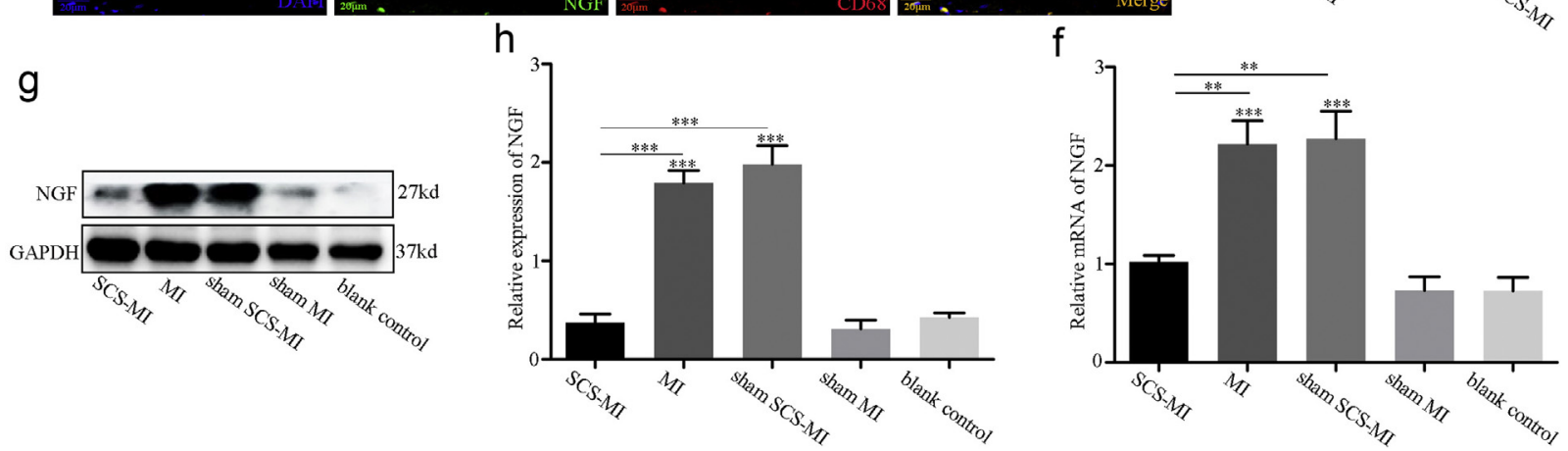

Figure 4. Effect of SCS on MI-induced inflammation. a. Representative immunohistochemistry photomicrographs of CD68 in the peri-infarct area two weeks after MI from the five groups mentioned previously. Three random fields of view were counted per section; two sections were evaluated from each heart, and three hearts were evaluated from each group. b. Quantitative analysis of (a) c-f. Levels of TNF- $a$, IL-6, IL-1 $\beta$, and NGF in the peri-infarct area by RT-PCR. g. NGF levels measured using Western blot. h. Quantitative analysis of (g) i. Representative confocal laser image of NGF and CD68 co-localization. TNF-a, tumor necrosis factor alpha; IL-6, interleukin-6; IL-1 $\beta$, interleukin-1 $\beta$; NGF, nerve growth factor; Ml, myocardial infarction; RT-PCR, reverse transcription polymerase chain reaction. Data from three independent experiments were presented as means \pm standard deviation. One-way ANOVA followed by Tukey's multiple comparisons test were used for multiplegroup comparisons $\left({ }^{*} p<0.05,{ }^{* *} p<0.01,{ }^{* *} p<0.001\right)$. [Color figure can be viewed at www.neuromodulationjournal.org]

Ischemic heart disease leads to ANS disorders. ${ }^{26}$ The correction of abnormal sympathetic and parasympathetic activity is important for improvement after ischemic heart disease. Neuromodulation using SCS has benefits for ischemic heart disease. As mentioned previously, SCS reduced the number of episodes of angina pectoris and need for short-acting nitrate tablets in previous studies. SCS also improved myocardial ischemic tolerance and perfusion reserve in patients with angina pectoris., 10,27,28 For patients with severe $\mathrm{HF}$, thoracic SCS improved symptoms, left ventricular function, and remodeling. $^{11,12}$ In canine and porcine ischemic models, SCS improved ventricular function and myocardial oxygen consumption and prevented ventricular arrhythmias. ${ }^{29-32}$

Previous studies have suggested that SCS exerts its cardiac protective effects by regulation of multiple levels of the ANS in ischemic hearts. First, afferent sensory transduction of ischemic myocardium results in an amplification of reflex efferent sympathoexcitation, and SCS attenuates this feedback control. ${ }^{17,18,33,34}$ Furthermore, evidence shows that plasma NE level is related to the frequency of sympathetic firing. ${ }^{35}$ Consistent with these studies, our results showed SCS attenuated the Ml-induced increase in 
a

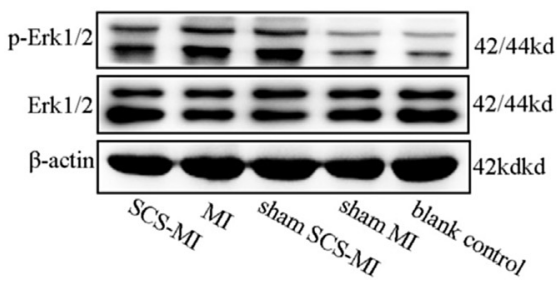

C

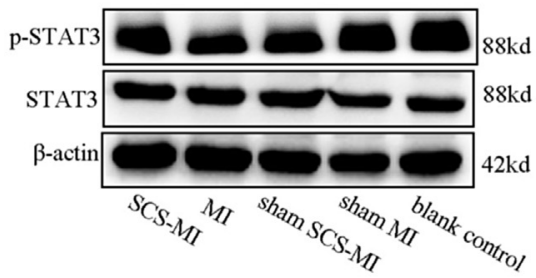

b

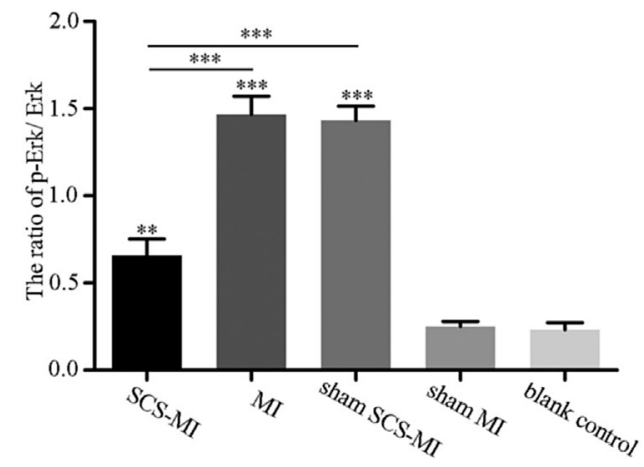

d

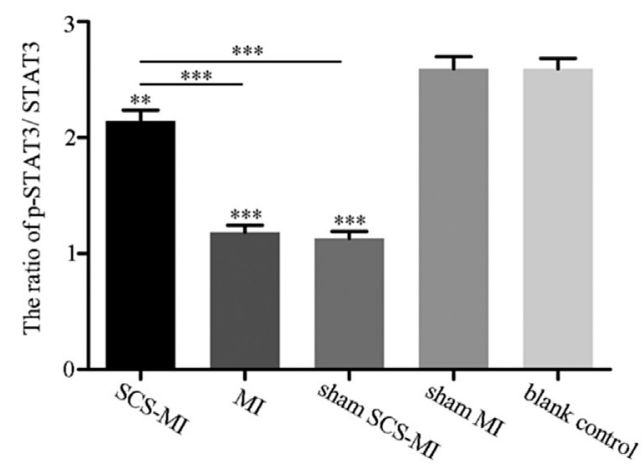

Figure 5. Effects of SCS on the Erk and STAT3 phosphorylation. a. Levels of phosphorylated-Erk, Erk, phosphorylated-STAT3, STAT3, and GAPDH in the peri-infarct area two weeks after MI from the 5 groups evaluated using Western blot. b. Quantitative analysis of the ratio of phosphorylated-Erk to total Erk. c. Quantitative analysis of the ratio of phosphorylated-STAT3 to total STAT3. Data from three independent experiments were presented as means \pm standard deviation. One-way ANOVA followed by Tukey's multiple comparisons test were used for multiple-group comparisons $\left({ }^{*} p<0.05,{ }^{* *} p<0.01,{ }^{* * *} p<0.001\right)$.

plasma NE and E levels, indicating that the hypothalamic-pituitaryadrenal axis might be involved in the regulation of systemic autonomic activity by SCS. Then, SCS inhibits the remodeling of regional cardiac sympathetic innervation in the peri-infarct and infarct regions of ischemic porcine models. ${ }^{15,36}$ Our results also showed that TH- and GAP43-positive nerve fibers increased in the MI group, and this increase was attenuated by SCS. This also might be partly due to SCS inhibiting the afferent sensory signals and efferent sympathoexcitation of ischemic myocardium. Besides, SCS inhibits the left stellate ganglion neural activity in a canine model of MI. ${ }^{16}$ Interestingly, cardiac NE decreased after MI, consistent with the previous studies, ${ }^{37}$ and might result from stress-induced regional NE depletion, ${ }^{38}$ decreased NE synthesis, ${ }^{39}$ or reduced NE reuptake. ${ }^{40}$ Recent studies have indicated that the transient cholinergic transdifferentiation after $\mathrm{MI}$ contributes to the reduced cardiac NE levels. ${ }^{41}$ Moreover, Ml-induced reduction in cardiac NE level was not affected by SCS, like previous studies, ${ }^{42}$ indicating that SCS probably mainly decreased systemic sympathetic activity, so may benefit ischemic hearts.

Importantly, in our study, SCS increased the plasma Ach levels and reduced plasma NE and $E$ levels, thereby reducing the abnormal systemic sympathetic and parasympathetic activity after MI. We also observed that Ml decreased the density of ChATpositive fibers, and the potential reason might relate to sympathetic activation and centrally mediated decline in parasympathetic activity in the cardiac ganglion after Ml. ${ }^{26,43,44}$ Previous studies have demonstrated a decrease in the proportion of neurons that receive afferent parasympathetic signals in the cardiac ganglion.
Therefore, the parasympathetic activity decreases, despite an intact neural network after $\mathrm{MI}^{45}$ However, in the present study, there was no difference for cardiac Ach level between the MI group and the blank control group. The possible reasons for this discrepancy might be due to transient cholinergic transdifferentiation ${ }^{37,41,46}$ and synthesis of non-neuronal Ach such as ischemic damaged cardiomyocytes $^{47,48}$ and some immune cells. ${ }^{49-51}$ The increase in the density of ChAT-positive fibers and cardiac Ach levels by SCS alluded that SCS might reduce the decline in central parasympathetic activity in the cardiac ganglion by increasing the use of intact but possibly not fully utilized neurons.

The ANS is a powerful regulator of the immune system. ${ }^{52-55}$ Sympathetic denervation, such as renal denervation, attenuated the inflammatory response in animal models of ischemic heart disease. $^{56,57}$ Vagus nerve stimulation inhibited the release of proinflammatory cytokines by the macrophages through the interaction of Ach and a7 nicotinic acetylcholine receptors (called the cholinergic anti-inflammatory pathway). ${ }^{58,59}$ This finding has been confirmed in several other studies. ${ }^{60,61}$ Therefore, we speculated that SCS may modulate the excessive inflammatory response after MI. Our results demonstrated that SCS downregulated macrophage infiltration and expression of inflammatory cytokines (TNF- $a$, IL-1 $\beta$, IL-6, and NGF) in rabbit hearts after MI, suggesting an anti-inflammatory effect of SCS. Previous studies have reported an important role of Erk and STAT3 pathways in the regulation of inflammatory factors production by the ANS. NE promoted the production of inflammatory factors through Erk phosphorylation. ${ }^{62}$ In contrast, activation of the $a-7$ subunit of the nicotinic 
a

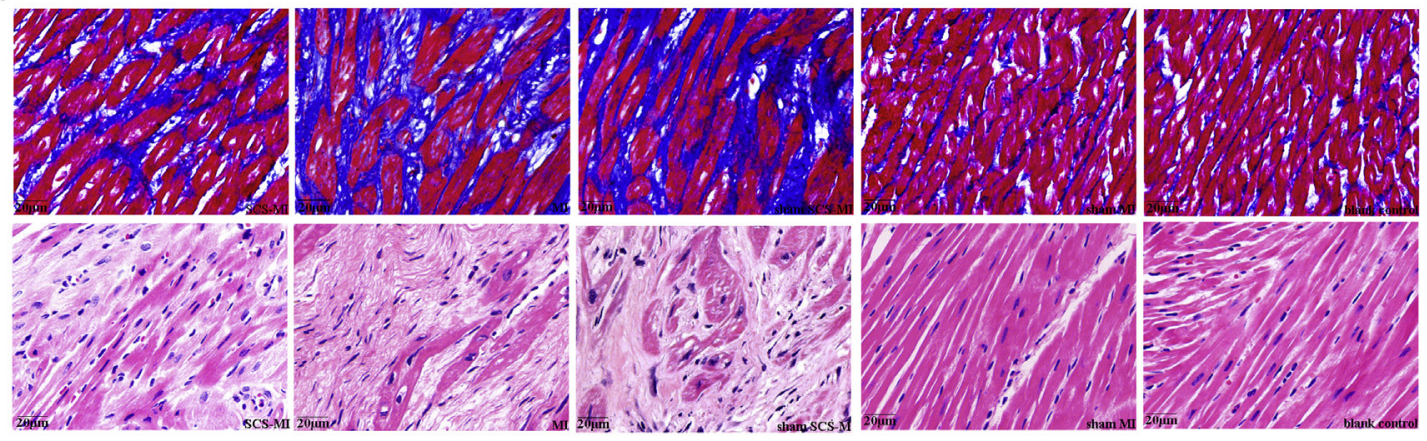

b

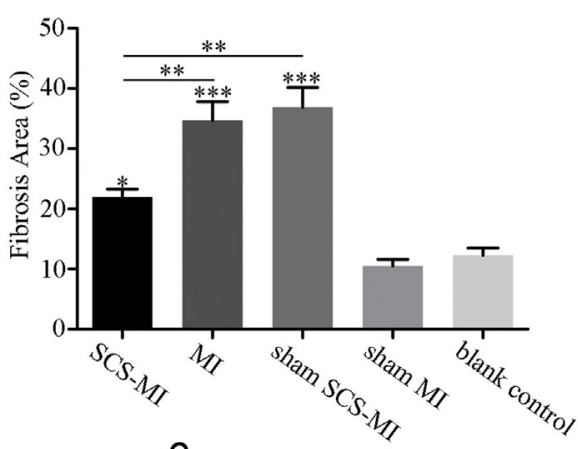

C

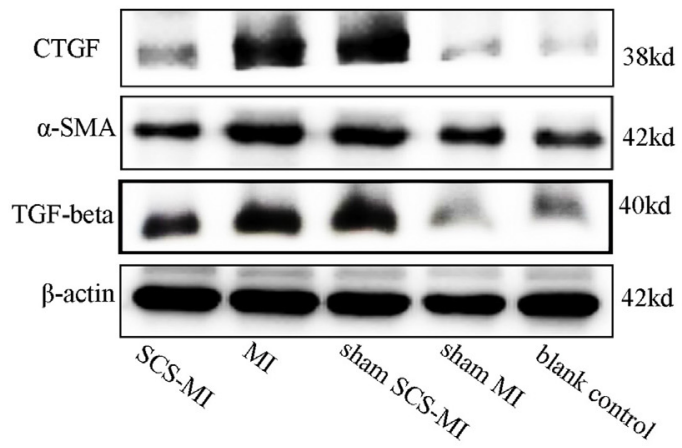

d

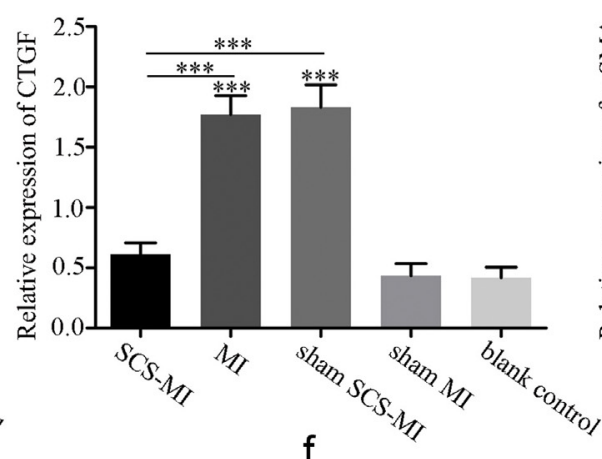

f

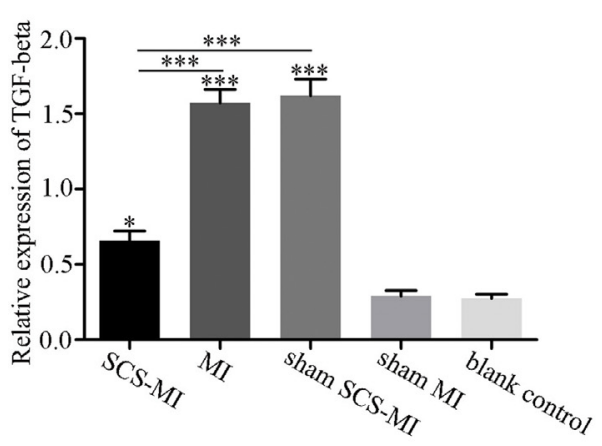

e

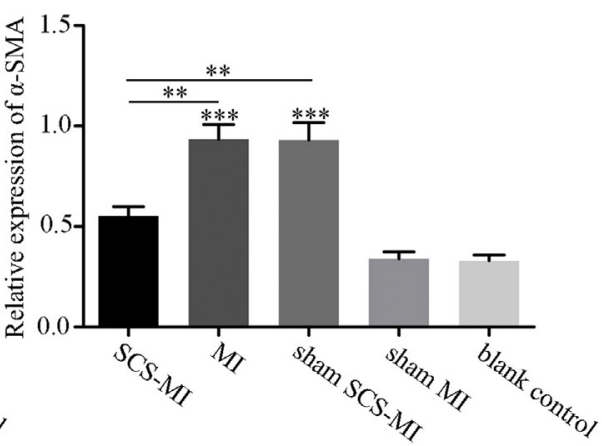

Figure 6. Effects of SCS on MI-induced myocardial fibrosis. a. Masson-trichrome and hematoxylin and eosin staining of the heart sections. b. Quantitative analysis of the proportional collagen area on trichrome-stained sections of the rabbit hearts. c. Levels of CTGF, $\alpha-S M A$, and TGF- $\beta$ in the rabbit hearts evaluated using Western blot. $d-f$. Quantitative analysis of (c). CTGF, connective tissue growth factor; $\alpha$-SMA, alpha-smooth muscle actin; TGF- $\beta$, transforming growth factor- $\beta$. Data from three independent experiments were presented as means \pm standard deviation. One-way ANOVA followed by Tukey's multiple comparisons test were used for multiplegroup comparisons $\left({ }^{*} p<0.05,{ }^{* *} p<0.01,{ }^{* * *} p<0.001\right)$. [Color figure can be viewed at www.neuromodulationjournal.org]

acetylcholine receptor inhibited Erk phosphorylation, thereby reducing the inflammatory response. ${ }^{63}$ Ach inhibited the inflammatory response by increasing the Jak2/STAT3 signaling cascade through the activation of the a-7 subunit of the nicotinic acetylcholine receptor. ${ }^{64} \mathrm{NE}$ reuptake inhibitors alleviated the inflammatory response through the modulation of the STAT3/IL-17/ RANKL axis. ${ }^{65}$ In our in vitro experiments, we used varying concentrations $(0,0.01,0.1$, or $1 \mu \mathrm{M})$ of NE to process RAW264.7 cells with or without LPS $(1 \mu \mathrm{g} / \mathrm{ml})$ activation. Our results showed that NE effectively stimulated the LPS-stimulated macrophages to express TNF- $\alpha$, IL-6, IL-1 $\beta$, and NGF in a dose-dependent manner. However, NE had no effect on RAW264.7 cells without LPS activation (Fig. S1). Erk inhibitors and STAT3 activators alleviated the expression of TNF- $a$, IL-6, IL-1 $\beta$, and NGF (Fig. S2). SCS might exert its anti-inflammatory effects through a similar mechanism. In our study, the ratio of phosphorylated-Erk to total Erk was higher and the ratio of phosphorylated-STAT3 to total STAT3 was lower in the $\mathrm{Ml}$ group than in the blank control group. These changes were attenuated by SCS and may be responsible for its anti-inflammatory effects.

Ventricular fibrosis is common after ischemic heart disease. Ventricular fibrosis increases the formation of critical reentry pathways, ${ }^{66}$ predisposing to cardiac structural remodeling. ${ }^{67}$ Therefore, researchers have proposed that ventricular fibrosis after $\mathrm{Ml}$ is a risk factor for arrhythmias and sudden cardiac death. ${ }^{68,69}$ Consequently, the inhibition of fibrosis may improve the prognosis after Ml by preventing Ml-induced malignant ventricular arrhythmias and HF. In the present study, SCS reduced the collagen 
area and inhibited the activation of myocardial fibrosis, which may be responsible for the protective effect of SCS after MI.

Our study had several limitations. First, we did not study the relative contributions of the sympathetic and parasympathetic systems to the effects of SCS on autonomic remodeling. Second, the mechanisms of effects of SCS on inflammation were not studied. Further animal studies are needed to evaluate the mechanism of SCS effects on inflammation by inhibiting Erk and activating or inhibiting STAT3. Third, we did not study the mechanisms of effects of SCS on MI-induced myocardial fibrosis, which should be addressed in future studies.

\section{CONCLUSIONS}

SCS improved the abnormal sympathetic and parasympathetic activity after MI. In addition, SCS inhibited inflammation, increased STAT3, reduced Erk, and prevented myocardial fibrosis. Our results provide a basis for future research of biological mechanisms of SCS in the treatment for MI.

\section{Authorship Statement}

Yuxian He, Zewei Sun, and Jiajia Jiang designed and conducted the study, including experimental design, animal model construction, and molecular testing. Xiang Yin and Jie Han analyzed the data. Yuxian He, Zewei Sun, and Yuanyuan Zhang prepared the manuscript draft. Yuxian He and Zewei Sun revised the manuscript. We would like to thank Dr. Zheng for his editorial guidance during preparation of this manuscript. All authors approved the final version of the manuscript.

\section{How to Cite This Article:}

He Y., Sun Z., Jiang J., Yin X., Han J., Zhang Y., Zheng L. 2023. Spinal Cord Stimulation Attenuates Neural Remodeling, Inflammation, and Fibrosis After Myocardial Infarction.

Neuromodulation 2023; 26: 57-67.

\section{SUPPLEMENTARY DATA}

To access the supplementary material accompanying this article, visit the online version of Neuromodulation: Technology at the Neural Interface at www.neuromodulationjournal.org and at https:// doi.org/10.1016/j.neurom.2021.09.005.

\section{REFERENCES}

1. Timmis A, Townsend N, Gale C, et al. European Society of Cardiology: cardiovascular disease Statistics 2017. Eur Heart J. 2018;39:508-579.

2. Sulo G, Igland J, Vollset SE, et al. Trends in incident acute myocardial infarction in Norway: an updated analysis to 2014 using national data from the CVDNOR project. Eur J Prev Cardiol. 2018;25:1031-1039.

3. Johansson S, Rosengren A, Young K, Jennings E. Mortality and morbidity trends after the first year in survivors of acute myocardial infarction: a systematic review. BMC Cardiovasc Disord. 2017;17:53.

4. Herring N, Kalla M, Paterson DJ. The autonomic nervous system and cardiac arrhythmias: current concepts and emerging therapies. Nat Rev Cardiol. 2019;16:707-726.
5. Goldberger JJ, Arora R, Buckley U, Shivkumar K. Autonomic nervous system dysfunction: JACC focus seminar. J Am Coll Cardiol. 2019;73:1189-1206.

6. Al-Kaisy A, Van Buyten JP, Kapural L, et al. $10 \mathrm{kHz}$ spinal cord stimulation for the treatment of non-surgical refractory back pain: subanalysis of pooled data from two prospective studies. Anaesthesia. 2020;75:775-784.

7. Kapural L, Yu C, Doust MW, et al. Novel 10-kHz high-frequency therapy (HF10 therapy) is superior to traditional low-frequency spinal cord stimulation for the treatment of chronic back and leg pain: the SENZA-RCT randomized controlled trial. Anesthesiology. 2015;123:851-860.

8. Deer TR, Mekhail N, Provenzano D, et al. The appropriate use of neurostimulation of the spinal cord and peripheral nervous system for the treatment of chronic pain and ischemic diseases: the Neuromodulation Appropriateness Consensus Committee. Neuromodulation. 2014;17:515-550; discussion 550.

9. Lanza GA, Barone L, Di Monaco A. Effect of spinal cord stimulation in patients with refractory angina: evidence from observational studies. Neuromodulation. 2012;15:542-549; discussion 549.

10. Saraste $A$, Ukkonen $H$, Varis $A$, et al. Effect of spinal cord stimulation on myocardial perfusion reserve in patients with refractory angina pectoris. Eur Heart J CardiovasC Imaging. 2015;16:449-455.

11. Tse HF, Turner S, Sanders $P$, et al. Thoracic Spinal Cord Stimulation for Heart Failure as a Restorative Treatment (SCS HEART study): first-in-man experience. Heart Rhythm. 2015;12:588-595.

12. Torre-Amione G, Alo K, Estep JD, et al. Spinal cord stimulation is safe and feasible in patients with advanced heart failure: early clinical experience. Eur J Heart Fail. 2014;16:788-795.

13. Zipes DP, Neuzil P, Theres $H$, et al. Determining the feasibility of spinal cord neuromodulation for the treatment of chronic systolic heart failure: the DEFEAT-HF study. JACC Heart Fail. 2016;4:129-136.

14. Upadhyay GA, Singh JP. Spinal cord stimulation for heart failure in the DEFEAT-HF study: lost battle or lasting opportunities? JACC Heart Fail. 2016:4:137-139.

15. Liao SY, Liu Y, Zuo M, et al. Remodelling of cardiac sympathetic re-innervation with thoracic spinal cord stimulation improves left ventricular function in a porcine model of heart failure. Europace. 2015:17:1875-1883.

16. Wang S, Zhou X, Huang B, et al. Spinal cord stimulation protects against ventricular arrhythmias by suppressing left stellate ganglion neural activity in an acute myocardial infarction canine model. Heart Rhythm. 2015;12:1628-1635.

17. Salavatian S, Ardell SM, Hammer M, Gibbons D, Armour JA, Ardell JL. Thoracic spinal cord neuromodulation obtunds dorsal root ganglion afferent neurona transduction of the ischemic ventricle. Am J Physiol Heart Circ Physiol 2019:317:H1134-H1141.

18. Salavatian S, Beaumont E, Gibbons D, et al. Thoracic spinal cord and cervical vagosympathetic neuromodulation obtund nodose sensory transduction of myocardial ischemia. Auton Neurosci. 2017:208:57-65.

19. Horn CC, Ardell JL, Fisher LE. Electroceutical targeting of the autonomic nervous system. Physiology (Bethesda). 2019;34:150-162.

20. Lopshire JC, Zipes DP. Spinal cord stimulation for heart failure: preclinical studies to determine optimal stimulation parameters for clinical efficacy. J Cardiovas Transl Res. 2014;7:321-329.

21. Chang PC, Wo HT, Lee HL, et al. Sacubitril/Valsartan therapy ameliorates ventricular tachyarrhythmia inducibility in a rabbit myocardial infarction model. J Card Fail. 2020;26:527-537.

22. Sun Z, Han J, Zhao W, et al. TRPV1 activation exacerbates hypoxia/reoxygenationinduced apoptosis in $\mathrm{H} 9 \mathrm{C} 2$ cells via calcium overload and mitochondria dysfunction. Int J Mol Sci. 2014;15:18362-18380.

23. Kido M, Du L, Sullivan CC, et al. Hypoxia-inducible factor 1-alpha reduces infarction and attenuates progression of cardiac dysfunction after myocardial infarction in the mouse. J Am Coll Cardiol. 2005;46:2116-2124.

24. Schmittgen TD, Livak KJ. Analyzing real-time PCR data by the comparative $C(T)$ method. Nat Protoc. 2008;3:1101-1108.

25. Huang W, Zhang D, Millard RW, et al. Gene manipulated peritoneal cell patch repairs infarcted myocardium. J Mol Cell Cardiol. 2010;48:702-712.

26. Rajendran PS, Nakamura K, Ajijola OA, et al. Myocardial infarction induces structural and functional remodelling of the intrinsic cardiac nervous system. J Physiol. 2016;594:321-341.

27. de Jongste MJ, Hautvast RW, Hillege HL, Lie KI. Efficacy of spinal cord stimulation as adjuvant therapy for intractable angina pectoris: a prospective, randomized clinical study. Working Group on Neurocardiology. J Am Coll Cardiol. 1994:23:15921597.

28. Mannheimer C, Eliasson T, Andersson B, et al. Effects of spinal cord stimulation in angina pectoris induced by pacing and possible mechanisms of action. BMJ. 1993;307:477-480

29. Lopshire JC, Zhou X, Dusa C, et al. Spinal cord stimulation improves ventricular function and reduces ventricular arrhythmias in a canine postinfarction heart failure model. Circulation. 2009;120:286-294.

30. Issa ZF, Zhou X, Ujhelyi MR, et al. Thoracic spinal cord stimulation reduces the risk of ischemic ventricular arrhythmias in a postinfarction heart failure canine model. Circulation. 2005;111:3217-3220.

31. Liu Y, Yue WS, Liao SY, et al. Thoracic spinal cord stimulation improves cardiac contractile function and myocardial oxygen consumption in a porcine model of ischemic heart failure. J Cardiovasc Electrophysiol. 2012;23:534-540.

32. Odenstedt J, Linderoth $B$, Bergfeldt $L$, et al. Spinal cord stimulation effects on myocardial ischemia, infarct size, ventricular arrhythmia, and noninvasive 
electrophysiology in a porcine ischemia-reperfusion model. Heart Rhythm. 2011;8:892-898.

33. Ardell JL, Cardinal R, Vermeulen M, Armour JA. Dorsal spinal cord stimulation obtunds the capacity of intrathoracic extracardiac neurons to transduce myocardial ischemia. Am J Physiol Regul Integr Comp Physiol. 2009;297:R470R477.

34. Ding $X$, Ardell $J L$, Hua $F$, et al. Modulation of cardiac ischemia-sensitive afferent neuron signaling by preemptive $C 2$ spinal cord stimulation: effect on substance $P$ release from rat spinal cord. Am J Physiol Regul Integr Comp Physiol. 2008;294:R93R101.

35. Hasking GJ, Esler MD, Jennings GL, Burton D, Johns JA, Korner PI. Norepinephrine spillover to plasma in patients with congestive heart failure: evidence of increased overall and cardiorenal sympathetic nervous activity. Circulation. 1986;73:615-621.

36. Howard-Quijano K, Takamiya T, Dale EA, et al. Spinal cord stimulation reduces ventricular arrhythmias during acute ischemia by attenuation of regional myocardial excitability. Am J Physiol Heart Circ Physiol. 2017;313:H421-H431.

37. Kanazawa $H$, leda $M$, Kimura $K$, et al. Heart failure causes cholinergic transdifferentiation of cardiac sympathetic nerves via gp130-signaling cytokines in rodents. J Clin Invest. 2010;120:408-421.

38. Chidsey CA, Kaiser GA, Sonnenblick EH, Spann JF, Braunwald E. Cardiac norephinephrine stores in experimental heart failure in the dog. J Clin Invest. 1964:43:2386-2393.

39. Kimura $K$, leda $M$, Kanazawa $H$, et al. Cardiac sympathetic rejuvenation: a link between nerve function and cardiac hypertrophy. Circ Res. 2007;100: 1755-1764.

40. Merlet $P$, Valette $H$, Dubois-Randé JL, et al. Prognostic value of cardiac metaiodobenzylguanidine imaging in patients with heart failure. J Nucl Med. 1992;33:471-477.

41. Olivas A, Gardner RT, Wang L, Ripplinger CM, Woodward WR, Habecker BA Myocardial infarction causes transient cholinergic transdifferentiation of cardiac sympathetic nerves via gp130. J Neurosci. 2016;36:479-488.

42. Norrsell H, Eliasson T, Mannheimer $\mathrm{C}$, et al. Effects of pacing-induced myocardial stress and spinal cord stimulation on whole body and cardiac norepinephrine spillover. Eur Heart J. 1997;18:1890-1896.

43. Billman GE. A comprehensive review and analysis of 25 years of data from an in vivo canine model of sudden cardiac death: implications for future antiarrhythmic drug development. Pharmacol Ther. 2006;111:808-835.

44. Vanoli E, De Ferrari GM, Stramba-Badiale M, Hull SS, Foreman RD, Schwartz PJ. Vagal stimulation and prevention of sudden death in conscious dogs with a healed myocardial infarction. Circ Res. 1991;68:1471-1481.

45. Vaseghi M, Salavatian S, Rajendran PS, et al. Parasympathetic dysfunction and antiarrhythmic effect of vagal nerve stimulation following myocardial infarction. JCl Insight. 2017;2.

46. Wang L, Olivas A, Francis Stuart SD, et al. Cardiac sympathetic nerve transdifferentiation reduces action potential heterogeneity after myocardial infarction. Am J Physiol Heart Circ Physiol. 2020;318:H558-H565.

47. Roy A, Fields WC, Rocha-Resende C, et al. Cardiomyocyte-secreted acetylcholine is required for maintenance of homeostasis in the heart. FASEB J. 2013;27: 5072-5082.

48. Rocha-Resende C, Roy A, Resende R, et al. Non-neuronal cholinergic machinery present in cardiomyocytes offsets hypertrophic signals. J Mol Cell Cardiol. 2012:53:206-216.

49. Cox MA, Bassi C, Saunders ME, et al. Beyond neurotransmission: acetylcholine in immunity and inflammation. J Intern Med. 2020;287:120-133.

50. Kanashiro A, Hiroki $\mathrm{CH}$, da Fonseca DM, et al. The role of neutrophils in neuroimmune modulation. Pharmacol Res. 2020;151:104580.

51. Wessler I, Kirkpatrick CJ, Racké K. Non-neuronal acetylcholine, a locally acting molecule, widely distributed in biological systems: expression and function in humans. Pharmacol Ther. 1998;77:59-79.

52. Brinkman DJ, Ten Hove AS, Vervoordeldonk MJ, Luyer MD, de Jonge WJ. Neuroimmune interactions in the gut and their significance for intestinal immunity. Cells. 2019;8.

53. Hoover DB. Cholinergic modulation of the immune system presents new approaches for treating inflammation. Pharmacol Ther. 2017;179:1-16.

54. Kox M, van Eijk LT, Zwaag J, et al. Voluntary activation of the sympathetic nervous system and attenuation of the innate immune response in humans. Proc Natl Acad Sci U S A. 2014;111:7379-7384.
55. Abboud FM, Harwani SC, Chapleau MW. Autonomic neural regulation of the immune system: implications for hypertension and cardiovascular disease. Hypertension. 2012;59:755-762.

56. Sun $X$, Wei $Z$, Li Y, et al. Renal denervation restrains the inflammatory response in myocardial ischemia-reperfusion injury. Basic Res Cardiol. 2020;115:15.

57. Ziegler KA, Ahles A, Wille T, Kerler J, Ramanujam D, Engelhardt S. Local sympathetic denervation attenuates myocardial inflammation and improves cardiac function after myocardial infarction in mice. Cardiovasc Res. 2018;114:291-299.

58. Bonaz B, Sinniger V, Pellissier S. Anti-inflammatory properties of the vagus nerve: potential therapeutic implications of vagus nerve stimulation. I Physiol. 2016;594:5781-5790.

59. Wang $\mathrm{H}$, Yu M, Ochani M, et al. Nicotinic acetylcholine receptor alpha7 subunit is an essential regulator of inflammation. Nature. 2003:421:384-388.

60. Guarini S, Altavilla D, Cainazzo MM, et al. Efferent vagal fibre stimulation blunts nuclear factor-kappaB activation and protects against hypovolemic hemorrhagic shock. Circulation. 2003;107:1189-1194.

61. Bernik TR, Friedman SG, Ochani $M$, et al. Pharmacological stimulation of the cholinergic antiinflammatory pathway. J Exp Med. 2002;195:781-788.

62. Huang JL, Zhang YL, Wang CC, et al. Enhanced phosphorylation of MAPKs by NE promotes TNF-a production by macrophage through a adrenergic receptor. Inflammation. 2012;35:527-534.

63. Liu L, Wu H, Cao Q, Guo Z, Ren A, Dai Q. Stimulation of Alpha7 nicotinic acetylcholine receptor attenuates nicotine-induced upregulation of MMP, MCP-1, and RANTES through modulating ERK1/2/AP-1 signaling pathway in RAW264.7 and MOVAS cells. Mediators Inflamm. 2017;2017:2401027.

64. Peña G, Cai B, Liu J, et al. Unphosphorylated STAT3 modulates alpha 7 nicotinic receptor signaling and cytokine production in sepsis. Eur J Immunol. 2010;40:25802589.

65. Kamel KM, Gad AM, Mansour SM, Safar MM, Fawzy HM. Venlafaxine alleviates complete Freund's adjuvant-induced arthritis in rats: modulation of STAT-3/IL-17/ RANKL axis. Life Sci. 2019;226:68-76.

66. Nguyen TP, Qu Z, Weiss JN. Cardiac fibrosis and arrhythmogenesis: the road to repair is paved with perils. J Mol Cell Cardiol. 2014;70:83-91.

67. Gao R, Shi H, Chang S, et al. The selective NLRP3-inflammasome inhibitor MCC950 reduces myocardial fibrosis and improves cardiac remodeling in a mouse model of myocardial infarction. Int Immunopharmacol. 2019;74:105575.

68. Disertori M, Masè M, Ravelli F. Myocardial fibrosis predicts ventricular tachyarrhythmias. Trends Cardiovasc Med. 2017;27:363-372.

69. Disertori M, Rigoni M, Pace N, et al. Myocardial fibrosis assessment by LGE is a powerful predictor of ventricular tachyarrhythmias in ischemic and nonischemic LV dysfunction: a meta-analysis. JACC Cardiovasc Imaging. 2016;9:1046-1055.

\section{COMMENT}

The authors have executed a controlled, mechanistic study with rabbits to further unravel the effects of electrical neuromodulation, making use of an implanted SCS, in an ischemic heart model. Following the creation of acute myocardial infarction, with thorough methods, such as immunohistochemistry, staining and blood sampling for neurohormones, inflammatory responses, cardiac fibrosis and neural remodelling were studied. The outcomes showed improvement of abnormal (intrinsic) autonomic activity and attenuation of inflammation and myocardial fibrosis, following spinal cord stimulation (SCS). In addition, the authors correlate their findings with the results of clinical studies. Therefore, their study may be considered translational, providing the reader with a better insight into the underlying mechanisms of SCS on ischemic events.

Michael J.L. DeJongste, MD, PhD Groningen, The Netherlands 